\title{
T-S Fuzzy Model Based $H$-Infinity Control for 7-DoF Automobile Electrohydraulic Active Suspension System
}

\author{
Chenyu Zhou, ${ }^{1}$ Qiang Yu, ${ }^{1}$ Xuan Zhao, ${ }^{2}$ and Guohua $\mathrm{Zhu}^{3}$ \\ ${ }^{1}$ School of Automobile Engineering, Chang'an University, Xian 710064, China \\ ${ }^{2}$ Laboratory of Engine and Automobile Performance, Chang'an University, Xi'an 710064, China \\ ${ }^{3}$ State Key Laboratory of Advanced Design and Manufacture for Vehicle Body, Hunan University, Changsha 410082, China
}

Correspondence should be addressed to Chenyu Zhou; feibode@hotmail.com

Received 24 March 2017; Accepted 12 July 2017; Published 20 August 2017

Academic Editor: Enrique Onieva

Copyright (C) 2017 Chenyu Zhou et al. This is an open access article distributed under the Creative Commons Attribution License, which permits unrestricted use, distribution, and reproduction in any medium, provided the original work is properly cited.

\begin{abstract}
This paper presents a double loop controller for a 7-DoF automobile electrohydraulic active suspension via T-S fuzzy modelling technique. The outer loop controller employs a modified $H$-infinity feedback control based on a T-S fuzzy model to provide the actuation force needed to ensure better riding comfort and handling stability. The resulting optimizing problem is transformed into a linear matrix inequalities solution issue associated with stability analysis, suspension stroke limit, and force constraints. Integrating these via parallel distributed compensation method, the feedback gains are derived to render the suspension performance dependent on the perturbation size and improve the efficiency of active suspensions. Adaptive Robust Control (ARC) is then adopted in the inner loop design to deal with uncertain nonlinearities and improve tracking accuracy. The validity of improvements attained from this controller is demonstrated by comparing with conventional Backstepping control and a passive suspension on a 7-DoF simulation example. It is shown that the T-S fuzzy model based controller can achieve favourable suspension performance and energy conservation under both mild and malevolent road inputs.
\end{abstract}

\section{Introduction}

Suspension systems are considered to be one of the most important components providing trade-offs between ride comfort and handling stability in automobile. However, the selection of constant suspension properties explicitly blocks improvements in suspension performance. This observation has accelerated active suspension research in recent years. Presently, active suspension research can be categorized by two aspects, the actuator modification and the controller improvement. With respect to actuators, in [1], a frequency dependent weighting filter is integrated with a $H_{\infty}$ controller to come up with a linear electromagnetic actuator. This kind of actuator has been researched extensively as it has the bandwidth of a full electric system $[2,3]$. However, the force supplied is constricted and limited for heavyduty vehicles. In view of the high power-to-weight ratio it provides, electrohydraulic active suspension has continued to be recognized as one of the most efficient technologies for both passengers as well as heavy-duty commercial vehicles. This has increased discussions on the design of controllers using electrohydraulic actuators [4].

These developments have raised an interesting question that while modelling electrohydraulic units, some nonlinear and uncertain terms arise causing controller design difficulties, especially when linear control is used. For instance, in [5], though both time and frequency domain analyses are carried out using a 7-DoF model, some uncertainties associated with the hydraulic system are ignored. In [6], an adaptive control is proposed while viewing the hydraulic system simply as a force output. However, under high frequency conditions, the system's performance degrades sharply owing to this simplification. Alley uses a pure PID controller to deal with nonlinearities [7] but finds it to be unsatisfactory. In order to overcome such problems, several robust nonlinear control techniques have been applied in active suspension control, for example, Backstepping and Slide Mode Control. Backstepping control is a conventional but expensive method 
based on the Lyapunov stability analysis [8]. An advantage with Backstepping control is that, notwithstanding the fact that its performance gets sharply degraded when the iterative steps number is very large or derivative explosion takes place, its robustness and asymptotical stability have made it popular in recent studies. From the viewpoint of Lyapunov function selection, it is demonstrated in [9] that the Barrier Lyapunov Function is capable of coming up with a less conservative initial value compared with the Quadratic Lyapunov Function. Slide Mode Control (SMC) can serve as another powerful strategy in overcoming unknown issues concerning nonlinearities as well as guaranteeing transient performance. A new method involving a disturbance observer with SMC is developed in [10] to help respond to disturbances better. However, instability appears in certain circumstances owing to delays and the appearance of chatter [11]. Subsequently, other methods involving complex learning mechanisms are proposed with a view for smoothing out such chattering. However, several complexities associated make their application difficult. In view of its superior robustness, adaptive robust control is introduced into electrohydraulic systems by Yao and Tomizuka [12]. By employing two terms, an adaptive term and a robustness term, the system could achieve an outstanding performance in terms of speed and accuracy performance. Subsequently, other researches enhance such improvements by according greater importance to actuator properties such as saturation and hard limits [13-15].

Another issue capturing the attention of researchers is concerned with contradiction between the objectives of minimum body acceleration and minimum suspension deflection. This leads to optimal control strategies seeking to achieve a balance between different optimization objectives. Despite the huge improvement effected through optimal control, the problem of explicitly adapting the weighting coefficients corresponding to different degrees of perturbations remained unsolved, especially in active suspension researches [16-19]. As a result, the energy efficiency of the active suspension controller is reduced and the algorithm turns out to be more conservative. In [20], dynamic programming is adopted to arrive at an online adaptive optimal controller. Though adaptive dynamic programming has been used extensively in optimal control theory, the persistence of partial system knowledge or model free algorithms continues to make stability analysis difficult. It is now clear that a model based control method is needed for facilitating the stability analysis of an active suspension. What is more, few attempts have been made to cover this research using a $7 \mathrm{DoF}$ model. This raises an interesting research challenge: How can we build an active suspension controller that satisfies different road-induced inputs while still facilitating stability analysis?

The fuzzy modelling method applied in active suspension system serves as a functional tool for improving the aforementioned performance. In light of the different and conflicting objectives, the overall system model can be obtained by fuzzy blending of weights included expressions. In this case, a number of subsystems are integrated in accordance with various requirements by using fuzzy IFTHEN rules that describe the system precisely. Reference [21] indicates this property in the frequency and time domains by assuming the body mass to be a fuzzy variable and taking the nonlinearities associated with the 2-DoF and 5-DoF models into consideration separately. The merits of this T-S fuzzy method lie in the easily analyzable model based theorem, which supplies a convenient tactic while conducting stability analysis for a linear subsystem.

This study adopts a T-S fuzzy scheme based on a 7-DoF active suspension model to determine the desired force for adaptive robust controller of the inner loop controller while tracking the force. The gains from this outer loop feedback controller can be derived effectively by solving a set of linear matrix inequalities related to three different objectives [2226]; these objectives subtly combine the stability analyses while improving the $H_{\infty}$ performance of the suspension system. As for the issue of suspension rattle space limit, it is necessary to take the suspension hard point into consideration and select it in forms of a complementary inequality. In accordance with actuation saturation, another indispensable inequality arising in our new method points to certain constraints on the actuation physical structure. Hence, in order to cover force tracking problem accurately, adaptive robust control is selected for the inner loop controller. In this method, the control output is designed as a combination of adaptive and robust terms, which link to performance in terms of speed and accuracy, respectively. Comparative simulations are used to demonstrate the effectiveness of the proposed approach. The results validate the effectiveness of the proposed approach and show the impressive complex performance of acceleration, rattle space, and robustness in the presence of uncertain nonlinearities.

The rest of this paper is organized as follows. In Section 2, the structure and the model of full car active suspension are presented. The T-S fuzzy based active suspension control is described in Section 3. Section 4 presents an adaptive robust control for the inner loop. Section 5 describes the design results and simulation experiment. Finally, Section 6 provides a summary of this study.

\section{Active Suspension Full Car Model}

As far as the attitude of full car is considered, a 7-DoF active suspension model is built. In contrast to earlier full car research, all the forces of active actuators are derived in this paper for each corner equation rather than for the center of mass.

As shown in Figure 1, $F_{s f r}, F_{s f l}, F_{s r r}$, and $F_{s r l}$ denote the spring elastic forces, $F_{d f r}, F_{d f l}, F_{d r r}$, and $F_{d r l}$ are the damper forces, and $u_{1}, u_{2}, u_{3}$, and $u_{4}$ denote the forces arising from active actuation. At each corner, $y_{s i}(i=$ $1,2,3,4)$ and $y_{i}(i=1,2,3,4)$ are the displacements of the sprung and unsprung masses, respectively. $y, \theta \varphi$ denote the displacement of the center of gravity, the pitch angle, and the roll angle, respectively. The disturbances arising from road irregularities at each corner are $y_{01}, y_{02}, y_{03}$, and $y_{04}$. The structural parameters $a, b, c$, and $d$ are explicitly shown in Figure 1. 


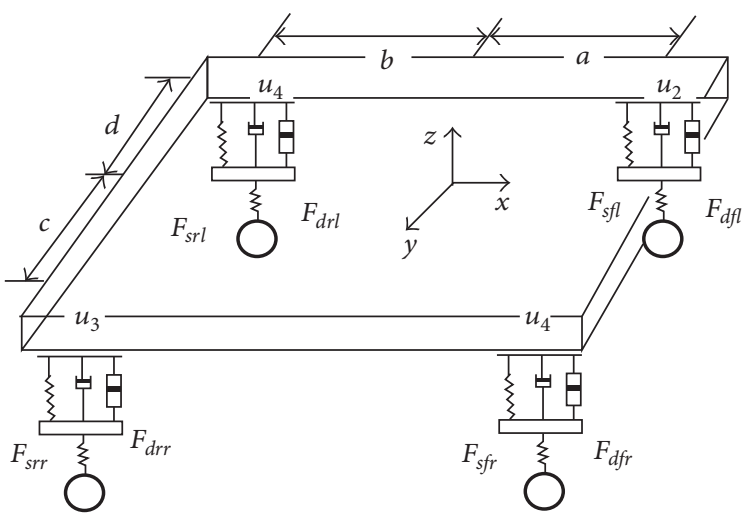

FIgURE 1: Active suspension structure for the 7-DoF full car model.

As to the physical structure of full car model, the kinematic relationship between different corners and the attitude of the car can be expressed as

$$
\begin{aligned}
& y_{s 1}=y+a \sin \theta-c \sin \varphi \\
& y_{s 2}=y+a \sin \theta+d \sin \varphi \\
& y_{s 3}=y-b \sin \theta-c \sin \varphi \\
& y_{s 4}=y-b \sin \theta+d \sin \varphi .
\end{aligned}
$$

The dynamical equations can then be expressed as

$$
\begin{aligned}
& m \ddot{y}=-F_{d f r}-F_{s f r}-F_{d f l}-F_{s f l}-F_{s r r}-F_{d r r}-F_{d r l} \\
& \quad-F_{s r l}+u_{1}+u_{2}+u_{3}+u_{4} \\
& I_{y y} \ddot{\theta}=\left[-a\left(F_{d f l}+F_{d f r}+F_{s f l}+F_{s f r}\right)\right. \\
& \left.\quad+b\left(F_{d r l}+F_{d r r}+F_{s r l}+F_{s r r}\right)\right]+a\left(u_{1}+u_{2}\right)-b\left(u_{3}\right. \\
& \left.\quad+u_{4}\right) \\
& I_{x x} \ddot{\varphi}=\left[-d\left(F_{d f l}+F_{d r l}+F_{s f l}+F_{s r l}\right)\right. \\
& \left.\quad+c\left(F_{d f r}+F_{d r r}+F_{s f r}+F_{s r r}\right)\right]-c\left(u_{1}+u_{3}\right)+d\left(u_{2}\right. \\
& \left.\quad+u_{4}\right),
\end{aligned}
$$

where $I_{y y}$ stands for the moment of inertia around the pitch axis and $I_{x x}$ is the roll axis moment of inertia.

All the sprung mass dynamics can be expressed more briefly in a matrix form such that the control force is produced in accordance with each corner:

$$
\begin{gathered}
{\left[\begin{array}{l}
\ddot{y}_{s 1} \\
\ddot{y}_{s 2} \\
\ddot{y}_{s 3} \\
\ddot{y}_{s 4}
\end{array}\right]=\mathbf{M}^{-1} \mathbf{\Omega} \mathbf{B}\left[\begin{array}{l}
\dot{y}_{s 1}-\dot{y}_{1} \\
\dot{y}_{s 2}-\dot{y}_{2} \\
\dot{y}_{s 3}-\dot{y}_{3} \\
\dot{y}_{s 4}-\dot{y}_{4}
\end{array}\right]+\mathbf{M}^{-1} \mathbf{\Omega} \mathbf{K}\left[\begin{array}{l}
y_{s 1}-y_{1} \\
y_{s 2}-y_{2} \\
y_{s 3}-y_{3} \\
y_{s 4}-y_{4}
\end{array}\right]} \\
-\mathbf{M}^{-1} \mathbf{\Omega}\left[\begin{array}{l}
u_{1} \\
u_{2} \\
u_{3} \\
u_{4}
\end{array}\right],
\end{gathered}
$$

where

$$
\begin{aligned}
& \mathbf{M}=\left[\begin{array}{llll}
m & & & \\
& m & & \\
& & m & \\
& & m
\end{array}\right], \\
& \boldsymbol{\Omega}=\left[\begin{array}{llll}
-\alpha & -\beta & -\gamma & -\delta \\
-\beta & -\xi & -\delta & -q \\
-\gamma & -\delta & -p & -n \\
-\delta & -q & -n & -\omega
\end{array}\right], \\
& \mathbf{B}=\left[\begin{array}{llll}
B_{f} & & & \\
& B_{f} & & \\
& & B_{r} & \\
& & & B_{r}
\end{array}\right]
\end{aligned}
$$

$$
\mathbf{K}=\left[\begin{array}{llll}
K_{f} & & & \\
& K_{f} & & \\
& & K_{r} & \\
& & & K_{r}
\end{array}\right] \text {. }
$$


Here, $m$ stands for the sprung mass, $B_{f}$ and $B_{r}$ denote the front and rear suspension damping, respectively, and $K_{f}$ and $K_{r}$ are the suspension stiffness values.

$$
\begin{aligned}
& \alpha=1+\frac{1}{I_{y y}} m a^{2}+\frac{1}{I_{x x}} m c^{2}, \\
& \beta=1+m a^{2} \frac{1}{I_{y y}}-m c d \frac{1}{I_{x x}}, \\
& \gamma=1-\frac{1}{I_{y y}} m a b+\frac{1}{I_{x x}} m c^{2}, \\
& \delta=1-m a b \frac{1}{I_{y y}}-m c d \frac{1}{I_{x x}}, \\
& \xi=1+\frac{1}{I_{y y}} m a^{2}+\frac{1}{I_{x x}} m d^{2}, \\
& q=1-m a b \frac{1}{I_{y y}}+m d^{2} \frac{1}{I_{x x}}, \\
& p=1+\frac{1}{I_{y y}} m b^{2}+\frac{1}{I_{x x}} m c^{2}, \\
& n=1+m b^{2} \frac{1}{I_{y y}}-m c d \frac{1}{I_{x x}}, \\
& w=1+m b^{2} \frac{1}{I_{y y}}+m d^{2} \frac{1}{I_{x x}} .
\end{aligned}
$$

Using the same method, we can convert the unsprung mass equation into

$$
\begin{gathered}
{\left[\begin{array}{l}
\ddot{y}_{1} \\
\ddot{y}_{2} \\
\ddot{y}_{3} \\
\ddot{y}_{4}
\end{array}\right]=\mathbf{M}_{\mathbf{u}}^{-1} \mathbf{B}\left[\begin{array}{l}
\dot{y}_{s 1}-\dot{y}_{1} \\
\dot{y}_{s 2}-\dot{y}_{2} \\
\dot{y}_{s 3}-\dot{y}_{3} \\
\dot{y}_{s 4}-\dot{y}_{4}
\end{array}\right]+\mathbf{M}_{\mathbf{u}}^{-1} \mathbf{K}\left[\begin{array}{l}
y_{s 1}-y_{1} \\
y_{s 2}-y_{2} \\
y_{s 3}-y_{3} \\
y_{s 4}-y_{4}
\end{array}\right]} \\
+\mathbf{M}_{\mathbf{u}}^{-1} \mathbf{K}_{\mathbf{t}}\left[\begin{array}{l}
y_{1}-y_{01} \\
y_{2}-y_{02} \\
y_{3}-y_{03} \\
y_{4}-y_{04}
\end{array}\right]-\mathbf{M}_{\mathbf{u}}^{-1} \mathbf{I}\left[\begin{array}{l}
u_{1} \\
u_{2} \\
u_{3} \\
u_{4}
\end{array}\right],
\end{gathered}
$$

where

$$
\begin{aligned}
\mathbf{M}_{\mathbf{u}} & =\left[\begin{array}{llll}
m_{1} & & & \\
& m_{2} & & \\
& & m_{3} & \\
& & & m_{4}
\end{array}\right], \\
\mathbf{K}_{\mathbf{t}} & =\left[\begin{array}{llll}
-k_{t 1} & & & \\
& -k_{t 2} & & \\
& & -k_{t 3} & \\
& & & -k_{t 4}
\end{array}\right],
\end{aligned}
$$

where I stands for the identity matrix for different dimensions. In these expressions, $m_{i}(i=1,2,3,4)$ is the unsprung mass while $k_{t i}(i=1,2,3,4)$ denotes the stiffness of the tyre.

In this research, we use a feedback control in which the state variables are selected as

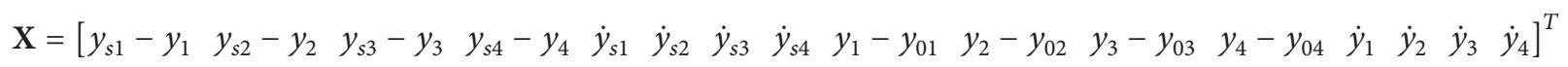

and the state equation can be derived as follows:

$$
\dot{\mathbf{X}}=\mathbf{A X}+\mathbf{B u}+\dot{E}_{\mathbf{0}},
$$

$$
\mathbf{E}=\left[\begin{array}{c}
\mathbf{O} \\
\mathbf{O} \\
-\mathbf{I} \\
\mathbf{O}
\end{array}\right],
$$

where

$$
\begin{aligned}
& A=\left[\begin{array}{cccc}
O & I & 0 & -I \\
M^{-1} \Omega K_{s} & M^{-1} \Omega B_{s} & 0 & -M^{-1} \Omega B_{s} \\
O & O & O & I \\
M_{u}^{-1} K_{s} & M_{u}^{-1} B_{s} & M_{u}^{-1} K_{t} & -M_{u}^{-1} B_{s}
\end{array}\right], \\
& \mathbf{B}=\left[\begin{array}{c}
O \\
-M^{-1} \Omega \\
O \\
-M_{u}^{-1}
\end{array}\right],
\end{aligned}
$$$$
\mathbf{u}=\left[\begin{array}{l}
u_{1} \\
u_{2} \\
u_{3} \\
u_{4}
\end{array}\right] \text {, }
$$$$
\dot{\mathbf{y}}_{\mathbf{0}}=\left[\begin{array}{c}
\dot{y}_{01} \\
\dot{y}_{02} \\
\dot{y}_{04} \\
\dot{y}_{04}
\end{array}\right] \text {. }
$$ 


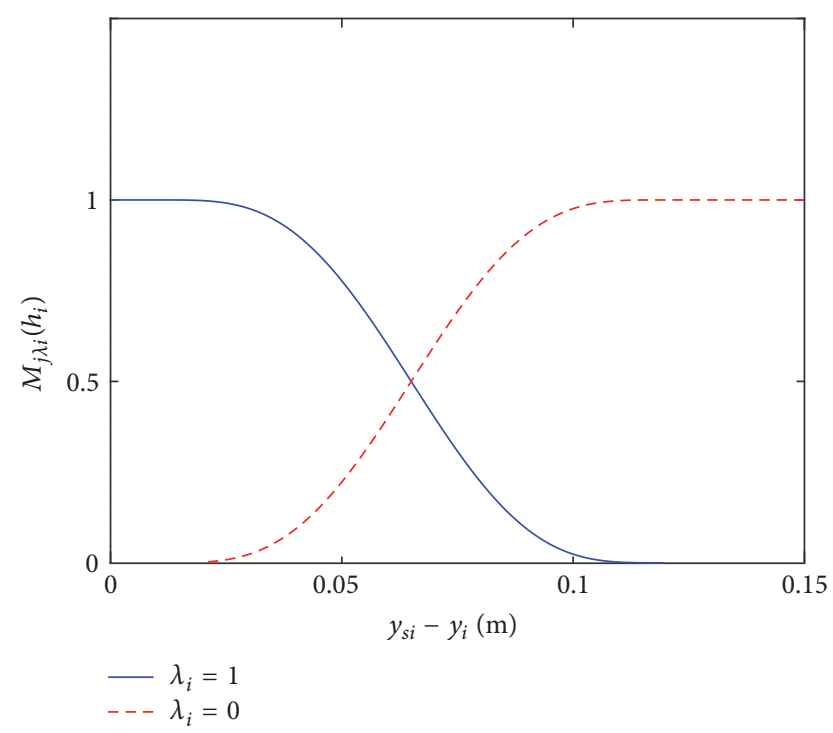

Figure 2: Fuzzy membership weight.

\section{Takagi-Sugeno Fuzzy Model Based Controller}

From the perspective of stability under all kinds of road disturbances, the local performance for each corner is deemed to preserve the suspension rattle space when the disturbance is large while the frequency is small and preserve the body acceleration under a small disturbance amplitude while the frequency is large, such that, in each corner, the performance matrix can be derived as

$$
\mathbf{e}_{\mathbf{i}}^{\mathbf{j}}=\left[\begin{array}{c}
\lambda_{i}^{j} \ddot{y}_{s i} \\
\left(1-\lambda_{i}^{j}\right)\left(y_{s i}-y_{i}\right) \\
u_{i}
\end{array}\right],
$$

where $\lambda_{i}^{j}$ is the weight according to the suspension dynamic deflection (it can be picked up from $\lambda_{i}^{j}=1$ or $\lambda_{i}^{j}=0$ ) and $\ddot{y}_{s i}$ and $\left(y_{s i}-y_{i}\right)(i=1,2,3,4)$ denote the acceleration and dynamic deflection at the specific corner, same as the control force input, $u_{i}$. Then, the performance parameters minimization issue which seems as the control objective is set to be realized.

Employing Takagi-Sugeno (T-S) model based control, a fuzzy membership function is designed according to the output performance weight, as shown in Figure 2.

Thus the 7-DoF full car model can be represented by a T-S fuzzy model composed of $16\left(2^{4}\right)$ rules as listed in Table 1.

Based on these fuzzy rules, the T-S fuzzy description defining the system dynamics and local output performance can be expressed as follows.

Model Rule 1.

If

$$
\begin{aligned}
& \lambda_{1}=1, \\
& \lambda_{1}=1,
\end{aligned}
$$

TABLE 1: List of fuzzy rules.

\begin{tabular}{lcccc}
\hline \multirow{2}{*}{ Rule number } & \multicolumn{4}{c}{ Premise variables } \\
& FR & FL & RR & RL \\
\hline 1 & 1 & 1 & 1 & 1 \\
2 & 1 & 1 & 1 & 0 \\
3 & 1 & 1 & 0 & 1 \\
4 & 1 & 1 & 0 & 0 \\
5 & 1 & 0 & 0 & 0 \\
6 & 1 & 0 & 0 & 1 \\
7 & 1 & 0 & 1 & 0 \\
8 & 1 & 0 & 1 & 1 \\
9 & 0 & 1 & 1 & 1 \\
10 & 0 & 1 & 1 & 0 \\
11 & 0 & 1 & 0 & 1 \\
12 & 0 & 1 & 0 & 0 \\
13 & 0 & 0 & 0 & 0 \\
14 & 0 & 0 & 0 & 1 \\
15 & 0 & 0 & 1 & 0 \\
16 & 0 & 0 & 1 & 1 \\
\hline
\end{tabular}

$$
\begin{aligned}
& \lambda_{3}=1, \\
& \lambda_{4}=1
\end{aligned}
$$

then

$$
\dot{\mathbf{X}}=\mathbf{A X}+\mathbf{B u}+\mathbf{E y}_{\mathbf{0}}
$$

and

$$
\mathbf{e}^{\mathbf{1}}=\left[\begin{array}{c}
\mathrm{F}_{11} \mathrm{~J}+\mathrm{F}_{11} \mathbf{M}^{-1} \Omega \mathrm{K}_{1} \\
\mathrm{~F}_{21} \sigma \\
\mathrm{K}_{1}
\end{array}\right] \mathrm{X},
$$

where

$$
\begin{aligned}
& \mathbf{F}_{11}=\left[\begin{array}{llll}
1 & & & \\
& 1 & & \\
& & 1 & \\
& & & 1
\end{array}\right], \\
& \mathbf{F}_{21}=\left[\begin{array}{llll}
0 & & & \\
& 0 & & \\
& & 0 & \\
& & & 0
\end{array}\right],
\end{aligned}
$$

$$
\begin{aligned}
\mathbf{J} & =\left[\begin{array}{llll}
\mathbf{M}^{-1} \boldsymbol{\Omega} \mathbf{K}_{\mathbf{s}} & \mathbf{M}^{-1} \boldsymbol{\Omega} \mathbf{B}_{\mathbf{s}} & \mathbf{O}_{4 \times 4} & -\mathbf{M}^{-1} \boldsymbol{\Omega} \mathbf{B}_{s}
\end{array}\right], \\
\boldsymbol{\sigma} & =\left[\begin{array}{ll}
\mathbf{I}_{4 \times 4} & \mathbf{0}_{4 \times 12}
\end{array}\right] .
\end{aligned}
$$


Model Rule j.

If

$$
\begin{aligned}
& \lambda_{1}=\lambda_{1}^{j}, \\
& \lambda_{2}=\lambda_{2}^{j}, \\
& \lambda_{3}=\lambda_{3}^{j}, \\
& \lambda_{4}=\lambda_{4}^{j}
\end{aligned}
$$

then

$$
\dot{\mathbf{X}}=\mathbf{A X}+\mathbf{B u}+\mathbf{E y}_{0}
$$

and

$$
\mathbf{e}^{j}=\left[\begin{array}{c}
\mathbf{F}_{1 j} \mathbf{J}+\mathbf{F}_{1 j} \mathbf{M}^{-1} \mathbf{\Omega} \mathbf{K}_{j} \\
\mathbf{F}_{2 j} \boldsymbol{\sigma} \\
\mathbf{K}_{j}
\end{array}\right] \mathbf{X},
$$

where

$$
\begin{aligned}
& \mathbf{F}_{1 j}=\left[\begin{array}{cccc}
\lambda_{1}^{j} & & & \\
& \lambda_{2}^{j} & & \\
& & \lambda_{3}^{j} & \\
& & & \lambda_{4}^{j}
\end{array}\right] \\
& \mathbf{F}_{2 j}=\left[\begin{array}{lrrr}
1-\lambda_{1}^{j} & & & \\
& 1-\lambda_{2}^{j} & & \\
& & 1-\lambda_{3}^{j} & \\
& & & 1-\lambda_{4}^{j}
\end{array}\right], \\
& \boldsymbol{\sigma}=\left[\begin{array}{ll}
\mathbf{I}_{4 \times 4} & \mathbf{0}_{4 \times 12}
\end{array}\right] .
\end{aligned}
$$

Model Rule 16.

If

$$
\begin{aligned}
& \lambda_{1}=0, \\
& \lambda_{2}=0, \\
& \lambda_{3}=1, \\
& \lambda_{4}=1
\end{aligned}
$$

then

$$
\dot{\mathbf{X}}=\mathbf{A X}+\mathbf{B u}+\mathbf{E y}_{\mathbf{0}}
$$

and

$$
\mathrm{e}^{16}=\left[\begin{array}{c}
\mathrm{F}_{116} \mathrm{~J}+\mathrm{F}_{116} \mathrm{M}^{-1} \Omega \mathrm{K}_{16} \\
\mathrm{~F}_{216} \sigma \\
\mathrm{K}_{16}
\end{array}\right] \mathrm{X}
$$

where

$$
\begin{aligned}
& \mathbf{F}_{116}=\left[\begin{array}{llll}
0 & & & \\
& & & \\
& 0 & & \\
& & 1 & \\
& & & 1
\end{array}\right] \text {, } \\
& \mathbf{F}_{216}=\left[\begin{array}{llll}
1 & & & \\
& 1 & & \\
& & 0 & \\
& & & 0
\end{array}\right], \\
& \boldsymbol{\sigma}=\left[\begin{array}{ll}
\mathbf{I}_{4 \times 4} & \mathbf{0}_{4 \times 12}
\end{array}\right] .
\end{aligned}
$$

The exact output performance model based on T-S fuzzy method can now be obtained as

$$
\begin{aligned}
e= & \sum_{j=1}^{16} M_{j \lambda 1}\left(h_{1}(t)\right) M_{j \lambda 2}\left(h_{2}(t)\right) M_{j \lambda 3}\left(h_{3}(t)\right) \\
& \cdot M_{j \lambda 4}\left(h_{4}(t)\right) \mathbf{e}^{\mathbf{j}}
\end{aligned}
$$

where $M_{j \lambda i}\left(h_{i}(t)\right)$ is the membership value of different corners when the dynamic deflection is assumed to be $h_{i}(t)$.

Here, we use a $H_{\infty}$ gain to modify the acceleration index such that a robust $H_{\infty}$ control is used in this research to keep the index bounded in a certain range responding to the road disturbance.

$$
\|L\|_{\infty}=\sup _{\left\|y_{0 i}\right\|_{2} \neq 0} \frac{\left\|\ddot{y}_{s i}\right\|_{2}}{\left\|\dot{y}_{0 i}\right\|_{2}} \quad(i=1,2,3,4)
$$

where $\left\|\ddot{y}_{s i}\right\|_{2}^{2}=\int_{0}^{\infty} \ddot{y}_{s i}^{T}(t) \ddot{y}_{s i}(t) d t$ and $\left\|\dot{y}_{0 i}\right\|_{2}^{2}=$ $\int_{0}^{\infty} \dot{y}_{0 i}^{T}(t) \dot{y}_{0 i}(t) d t$ are the norms in $L_{2}$ space and $\ddot{y}_{s i}, \dot{y}_{0 i}$ are the piecewise continuous, bounded functions. To solve this optimization problem, the elements from the fuzzy system performance (24) are assumed to be smaller than certain values as well as $L_{2}$ is minimized under the condition that the active suspension system is quadratically stable.

Next, the issue to design this controller is converted into a LMIs resolution problem that minimizes the $L_{2}$ gain subject to the LMIs (26), (27), and (28) under the fuzzy rule j: 


$$
\begin{aligned}
& {\left[\begin{array}{cccc}
\mathbf{Q A}^{T}+\mathbf{A Q}+\frac{1+\varepsilon}{2} \mathbf{Y}^{T} \mathbf{B}^{T}+\frac{1+\varepsilon}{2} \mathbf{B Y}+\kappa^{-1} \mathbf{B B}^{T} & \mathbf{Y}^{T} & \mathbf{Q}^{T} \boldsymbol{\psi}_{\mathbf{j i}}^{T} & \mathbf{E} \\
\mathbf{Y} & -\mathcal{\kappa}^{-1}\left(\frac{2}{1-\varepsilon}\right)^{2} \mathbf{I} & \mathbf{0} & \mathbf{0} \\
\boldsymbol{\psi}_{\mathrm{ji}} & \mathbf{0} & -\mathbf{I} & \mathbf{0} \\
\mathbf{E}^{T} & \mathbf{0} & \mathbf{0} & -0.25 \gamma^{2}
\end{array}\right]<\mathbf{0}} \\
& {\left[\begin{array}{cc}
-\mathbf{I} & \sqrt{\rho} \boldsymbol{\varphi}_{\mathrm{ji}} \mathbf{Q} \\
\sqrt{\rho} \mathbf{Q}^{T} \boldsymbol{\varphi}_{\mathrm{ji}}^{T} & -\delta_{j i}^{2} \mathbf{Q}
\end{array}\right]<\mathbf{0}} \\
& {\left[\begin{array}{cc}
-I & \sqrt{\rho} \boldsymbol{\Delta}_{\mathbf{j i}} \mathbf{Y} \\
\sqrt{\rho} \mathbf{Y}^{T} \boldsymbol{\Delta}_{\mathbf{j i}}^{T} & -\sigma_{j i}^{2} \mathbf{Q}
\end{array}\right]<\mathbf{0},}
\end{aligned}
$$

where $\mathbf{Q}=\mathbf{P}^{-1}, \mathbf{Y}=\mathbf{K} \mathbf{Q}$, and $\psi_{\mathbf{j i}(\mathbf{i}=1,2,3,4)} \mathbf{X}(\mathbf{t})$ is the first to the forth row in $\mathbf{e}^{\mathbf{j}}$ and denotes the different sprung mass acceleration at each corner and $\boldsymbol{\varphi}_{\mathrm{ji}(\mathrm{i}=1,2,3,4)} \mathbf{X}(\mathbf{t})$ denotes the suspension dynamic deflection in each corner which is simplified from the fifth to eighth row in $\mathbf{e}^{\mathbf{j}}$. The notation $\mathbf{M}>0(\mathbf{M}<0)$ denotes a positive matrix (negative matrix) for a real symmetric matrix $\mathbf{M}$.

$$
\begin{aligned}
\Delta_{\mathbf{j} 1} & =\left[\begin{array}{llll}
1 & 0 & 0 & 0 \\
0 & 0 & 0 & 0 \\
0 & 0 & 0 & 0 \\
0 & 0 & 0 & 0
\end{array}\right], \\
\Delta_{\mathbf{j} 2} & =\left[\begin{array}{llll}
0 & 0 & 0 & 0 \\
0 & 1 & 0 & 0 \\
0 & 0 & 0 & 0 \\
0 & 0 & 0 & 0
\end{array}\right], \\
\boldsymbol{\Delta}_{\mathbf{j} 3} & =\left[\begin{array}{llll}
0 & 0 & 0 & 0 \\
0 & 0 & 0 & 0 \\
0 & 0 & 1 & 0 \\
0 & 0 & 0 & 0
\end{array}\right], \\
\boldsymbol{\Delta}_{\mathbf{j} 4} & =\left[\begin{array}{llll}
0 & 0 & 0 & 0 \\
0 & 0 & 0 & 0 \\
0 & 0 & 0 & 0 \\
0 & 0 & 0 & 1
\end{array}\right] .
\end{aligned}
$$

As mentioned before, there would be 12 LMIs in each of the 16 different sub-fuzzy-system.

Proof. For the purpose of stability analysis, the Lyapunov function is captured as

$$
V=\mathbf{X}^{T} \mathbf{P X}
$$

where $\mathbf{P}$ is a positive matrix. The differential of this equation can be expressed as

$$
\begin{aligned}
\dot{V} & =\left(\mathbf{A X}(\mathbf{t})+\mathbf{E} \dot{\mathbf{y}}_{0}(\mathbf{t})+\mathbf{B} \frac{1+\varepsilon}{2} \mathbf{u}(\mathbf{t})+\mathbf{B v}(\mathbf{t})\right)^{T} \\
& \cdot \mathbf{P X}(\mathbf{t})+\mathbf{X}^{\mathrm{T}}(\mathbf{t}) \\
& \cdot \mathbf{P}\left(\mathbf{A X}(\mathbf{t})+\mathbf{E}_{\mathbf{0}}(\mathbf{t})+\mathbf{B} \frac{1+\varepsilon}{2} \mathbf{u}(\mathbf{t})+\mathbf{B v}(\mathbf{t})\right),
\end{aligned}
$$

where $\mathbf{v}(\mathbf{t})=\mathbf{u}(\mathbf{t})_{\text {saturation }}-(1+\varepsilon) \mathbf{u}(\mathbf{t}) / 2$.

Here we have used lemmas 1 and 2 derived from $[27,28]$.

Lemma 1. $\mathbf{u}(\mathbf{t})_{\text {saturation }}$ is defined as the saturation function of output force $\mathbf{u}(\mathbf{t})$, with the limit $\mathbf{u}(\mathbf{t})_{\max }$, as long as $|\mathbf{u}(\mathbf{t})| \leq$ $\mathbf{u}(\mathbf{t})_{\max } / \varepsilon$; then

$$
\left\|\mathbf{u}(\mathbf{t})_{\text {saturation }}-\frac{1+\varepsilon}{2} \mathbf{u}(\mathbf{t})\right\| \leq \frac{1-\varepsilon}{2}\|\mathbf{u}(\mathbf{t})\|
$$

and hence

$$
\begin{aligned}
\boldsymbol{\Omega}^{\mathrm{T}} \boldsymbol{\Omega} & \leq\left(\frac{1-\varepsilon}{2}\right)^{2} \mathbf{u}^{\mathrm{T}}(\mathbf{t}) \mathbf{u}(\mathbf{t}) \\
\boldsymbol{\Omega} & =\mathbf{u}(\mathbf{t})_{\text {saturation }}-\frac{1+\varepsilon}{2} u(t) \quad \text { where } 0<\varepsilon<1 .
\end{aligned}
$$

Lemma 2. For any appropriate matrix $S$ and $Z$, one has $S^{T} Z+Z^{T} S \leq \kappa S^{T} S+\kappa^{-1} Z^{T} Z$, where $\kappa>0$.

Then we can get

$$
\begin{aligned}
& \dot{V}(x(t)) \leq \mathbf{X}^{T}(\mathbf{t}) \\
& \cdot\left(\mathbf{A}^{T} \mathbf{P}+\frac{1+\varepsilon}{2} \mathbf{K}^{T} \mathbf{B}^{T} \mathbf{P}+\mathbf{P A}+\frac{1+\varepsilon}{2} \mathbf{P B K}\right) \mathbf{X}(\mathbf{t}) \\
& +\dot{\mathbf{y}}_{\mathbf{0}}^{T} \mathbf{E}^{T} \mathbf{P} \mathbf{X}+\mathbf{X}^{T} \mathbf{P E} \dot{y}_{\mathbf{0}}+\kappa\left(\frac{1-\varepsilon}{2}\right)^{2} \mathbf{u}^{T}(\mathbf{t}) \mathbf{u}(\mathbf{t}) \\
& +\kappa^{-1} \mathbf{X}^{T}(\mathbf{t}) \mathbf{P B B} \mathbf{B}^{T} \mathbf{P X}
\end{aligned}
$$


such that

$$
\begin{gathered}
\dot{V}(x(t)) \leq \mathbf{X}^{T}(\mathbf{t})\left(\mathbf{A}^{T} \mathbf{P}+\frac{1+\varepsilon}{2} \mathbf{K}^{T} \mathbf{B}^{T} \mathbf{P}+\mathbf{P A}\right. \\
\left.+\frac{1+\varepsilon}{2} \mathbf{P B K}+\kappa\left(\frac{1-\varepsilon}{2}\right)^{2} \mathbf{K}^{T} \mathbf{K}+\kappa^{-1} \mathbf{P B B} \mathbf{B}^{T} \mathbf{P}\right) \\
\cdot \mathbf{X}(\mathbf{t})+\dot{\mathbf{y}}_{\mathbf{0}}^{T} \mathbf{E}^{T} \mathbf{P X}+\mathbf{X}^{T} \mathbf{P} \mathbf{E} \dot{\mathbf{y}}_{\mathbf{0}} .
\end{gathered}
$$

To avoid redundant inequalities and efficiency reduction, the $L_{2}$ gain is chosen to integrate the suspension performance with stability analysis which can be defined as (25). To this end, the quadratic stability and the optimum $L_{2}$ gain can be obtained at the same time once the solution of this inequality is obtained.

Next, we assume the first middle term of right hand side as $\Theta$. Then, add $\mathbf{X}^{\mathrm{T}}(\mathbf{t}) \boldsymbol{\psi}_{\mathrm{ji}}^{T} \boldsymbol{\psi}_{\mathrm{ji}} \mathbf{X}(\mathbf{t})-\left(\gamma^{2} / 4\right) \dot{\mathbf{y}}_{0}^{T} \dot{\mathbf{y}}_{0}$ to both sides of this inequality to arrive at the following simplified form:

$$
\begin{gathered}
\dot{V}(x(t))+\mathbf{X}^{T}(\mathbf{t}) \psi_{\mathrm{ji}}^{T} \boldsymbol{\psi}_{\mathbf{j i}} \mathbf{X}(\mathbf{t})-\frac{\gamma^{2}}{4} \dot{\mathbf{y}}_{\mathbf{0}}^{T} \dot{\mathbf{y}}_{0} \\
\leq \mathbf{X}^{T}(\mathbf{t}) \Theta \mathbf{X}(\mathbf{t})+\dot{\mathbf{y}}_{\mathbf{0}}^{T} \mathbf{E}^{T} \mathbf{P X}+\mathbf{X}^{T} \mathbf{P E} \dot{\mathbf{y}}_{\mathbf{0}} \\
+\mathbf{X}^{T}(\mathbf{t}) \psi_{\mathbf{j i}}^{T} \psi_{\mathrm{ji}} \mathbf{X}(\mathbf{t})-\frac{\gamma^{2}}{4} \dot{\mathbf{y}}_{\mathbf{0}}^{T} \dot{\mathbf{y}}_{\mathbf{0}} .
\end{gathered}
$$

The system is indicated to be quadratically stable if the right hand side of (36) is less than zero, which can be simplified as

$$
\left[\begin{array}{cc}
\Theta+\boldsymbol{\psi}_{\mathrm{ji}}^{T} \psi_{\mathrm{ji}} & \mathbf{P E} \\
\mathbf{E}^{T} \mathbf{P} & -0.25 \gamma^{2}
\end{array}\right]<0 .
$$

Next, we pre- and postmultiply the above equation with $\operatorname{diag}(\mathbf{Q} \quad \mathbf{I})$ and its transpose where $\mathbf{Q}=\mathbf{P}^{-1}$ and use the Schur complement to make the inequality amenable to solution. The $H_{\infty}$ performance can be assessed in (38) using the initial condition $\mathbf{X}(\mathbf{0})=\mathbf{0}$ and $V(\mathbf{X}(\mathbf{0}))=0$.

$$
\begin{aligned}
& V(\mathbf{X}(\mathbf{t}))+\int_{0}^{t}\left\|\ddot{y}_{s i}(\tau)\right\|^{2} d \tau-\frac{\gamma^{2}}{4} \int_{0}^{t}\left\|\dot{\mathbf{y}}_{\mathbf{0}}(\boldsymbol{\tau})\right\|^{2} d \tau \\
& \quad \leq V(\mathbf{X}(\mathbf{0})) .
\end{aligned}
$$

Furthermore, assuming that the road input energy is bounded by $\dot{y}_{0 \max }$, an ellipsoid $\Omega(P, \rho)=\{x(t) \quad \mid$ $\left.\mathbf{X}^{T}(t) \mathbf{P}_{\mathbf{i}} \mathbf{X}(\mathbf{t}) \leq \rho\right\}$ can be specified by $\rho=\gamma^{2} / 4 \cdot \dot{y}_{0 \max }$. This means that, with the disturbance energy bounded by $\dot{y}_{0 \max }$, all reachable states are located within this ellipsoid set.

Employing the same algorithm at the other corners, we can confirm that all the corners hold constrained $H_{\infty}$ performance and make the controller stable. In the end, the description of this subsystem can be synthesized through the LMIs (26).

In response to the suspension rattle space, an inequality interaction with the second element of (11) is adopted as

$$
\left\|\varphi_{\mathrm{ji}} \mathbf{X}(\mathbf{t})\right\|_{2}^{2}<\delta^{2}
$$

so that

$$
\begin{aligned}
& \max \left|\left(y_{s i}(t)-y_{i}(t)\right)\right|^{2} \leq \max \left\|\mathbf{X}^{T}(\mathbf{t}) \boldsymbol{\varphi}_{\mathrm{ji}}^{T} \boldsymbol{\varphi}_{\mathrm{ji}} \mathbf{X}(\mathbf{t})\right\|_{2}^{2} \\
& \leq \rho \cdot \max \left(\operatorname{eigen}\left(\mathbf{P}_{\mathbf{j}}^{-1 / 2} \boldsymbol{\varphi}_{\mathrm{ji}}^{T} \boldsymbol{\varphi}_{\mathrm{ji}} \mathbf{P}_{\mathbf{j}}^{-1 / 2}\right)\right),
\end{aligned}
$$

where eigen $(\cdot)$ is the eigenvalue of the matrix.

The constraint in (39) is guaranteed on the condition that

$$
\rho \mathbf{P}_{\mathbf{j}}^{-1} \boldsymbol{\varphi}_{\mathbf{j i}}^{T} \boldsymbol{\varphi}_{\mathrm{ji}} \mathbf{P}_{\mathbf{j}}^{-1} \leq \delta^{2} \mathbf{P}_{\mathbf{j}}^{-1} .
$$

Similar to the acceleration at each corner, a constraint on the suspension dynamic deflection is applied at all the corners of the car. Using the Schur complement, the rattle space limit can be explicitly shown to be as in (27).

With regard to the force produced by the actuation, a force restraint is imposed on the control algorithm as far as the protection issue is considered. However, the unknown matrices, $\mathbf{Q}$ and $\mathbf{Y}$, are involved in the force presentation, which makes the LMI difficult to involve all the corners. To deal with this problem, an adjusted matrix, $\Delta_{\mathrm{ji}}$, is employed to figure out the control force at each corner. Same as before, the Schur complement is used here to make it into a matrix form, so as to arrive ultimately at the descriptions in (28).

Then, objective of this controller is transferred to find the resolution of the linear matrix inequalities (26), (27), and (28) and obtain the minimization of parameter $\gamma^{2}$. To attain minimum body acceleration, limited suspension rattle space, and restrained actuator force, a minimization issue, based on the fuzzy representation, is proposed as the local performance indicator to find the optimal feedback gains and to accommodate to the suspension requirements. If the LMIs are found to be feasible (which means that the coefficients $\mathbf{Q}, \mathbf{Y}$, and $\mathbf{P}$ are determinable), the system is assumed to be under quadratic stability and achieve a significantly better performance. Finally, we can obtain the feedback gain as $\mathbf{K}_{\mathbf{j}}=\mathbf{Y}_{\mathbf{j}} \mathbf{Q}_{\mathbf{j}}{ }^{-1}$ (j denotes the different fuzzy subsystems). After setting the force derived from $\mathbf{u}=$ $\mathbf{F}_{\mathbf{d}}=\sum_{j=1}^{16} M_{j \lambda 1}\left(h_{1}(t)\right) M_{j \lambda 2}\left(h_{2}(t)\right) M_{j \lambda 3}\left(h_{3}(t)\right) M_{j \lambda 4} \mathbf{K} \mathbf{j} \mathbf{X}(\mathbf{t})$ using the PDC method, the inner loop becomes available for further design.

\section{Adaptive Robust Control of Inner Loop}

In order to address the problem induced by uncertain nonlinearities in an electrohydraulic system, ARC is employed to track the desired force and to maintain stability under inconstant conditions at different corners. The electrohydraulic actuator can then be modelled as

$$
\begin{aligned}
\dot{F}_{a i} & =\theta\left[q_{1}\left(\dot{y}_{s i}-\dot{y}_{i}\right)+q_{2} g_{i}(t)\right]+k_{f}\left(\ddot{y}_{s i}-\ddot{y}_{i}\right) \\
g_{i}(t) & =x_{s i} \sqrt{P_{s}-\frac{\operatorname{sgn}\left(g_{i}(t)\right) F_{a i}}{A}},
\end{aligned}
$$

where $\theta$ is an uncertainty parameter, $q_{1}, q_{2}$, and $k_{f}$ are known parameters, $P_{s}$ is the system fluid pressure, $F_{a i}$ is the actual force imposed on the piston in the actuator, $A$ is the piston 
area, and $x_{s i}$ is the displacement of the spool. The subscript $i$ stands for the different corners of the full car model.

Next, the input term, $g_{i}(t)$, is assumed to be a combination of two parts, the speed term, $g_{a}(t)$, and the accuracy term, $g_{s}(t)$. Using the following Lemma 3 in $[29,30]$, we can now determine these two terms separately as (46).

Lemma 3. For the function

$$
f(u, x)=F(x)\{A(x) v+B(x) u\}
$$

where $A(x), B(x)$ are unknown functions, $F(x)$, v are known functions. If there exists $A_{M}(x)$ and $B_{M}(x)$ such that

$$
\begin{aligned}
|A(x)| & \leq\left|A_{M}(x)\right| \\
0 & <\frac{B_{m}(x)}{B(x)} \leq 1,
\end{aligned}
$$

then, for each $\varepsilon>0$, there exists $u(x)$ such that $f(u(x), x) \leq$ $\varepsilon v^{2}$ and one such function is

$$
u(x)=-F(x) \frac{1}{4 \varepsilon} \frac{A_{M}(x)^{2}}{B_{m}(x)} .
$$

Furthermore, $F(x) B(x) u(x) \leq 0$.

$$
\begin{aligned}
g_{a i} & =\frac{1}{q_{2}}\left\{-q_{1}\left(\dot{y}_{s i}-\dot{y}_{i}\right)\right. \\
& \left.+\frac{1}{\widehat{\theta}_{1 \pi}}\left[-k_{f}\left(\ddot{y}_{s i}-\ddot{y}_{i}\right)+\dot{F}_{d i}-c_{1} Z_{1}\right]\right\} \\
\dot{\hat{\theta}}_{\pi} & =\eta Z_{1}\left[q_{2} g_{a i}+q_{1}\left(\dot{y}_{s i}-\dot{y}_{i}\right)\right] \\
g_{s i} & =-Z_{1} \frac{1}{4 \theta_{m} q_{2}}\left\{\frac{1}{\varepsilon_{1}} \prod_{M}^{2}\left[q_{1}\left(\dot{y}_{s i}-\dot{y}_{i}\right)+q_{2} g_{a i}\right]^{2}\right\},
\end{aligned}
$$

where $\widehat{\theta}_{\pi}$ is the projection of $\theta$ and $\widetilde{\theta}_{\pi}=\theta-\widehat{\theta}_{\pi}, \eta$ is an adaptive parameter, and $\Pi_{M}^{2}$ is a positive number that ensures that $\left|\widetilde{\theta}_{\pi}\right|<\Pi_{M}, \theta_{m}$, and $\theta_{M}$ are the minimum and maximum values, respectively, of the unknown parameter $\theta . Z_{1}=F_{a i}-F_{d i}$ is the control objective.

Assuming that the desired force has been designed from the outer loop, this tracking issue can be dealt with using the inner loop ARC method. In the interest of simplicity, the processes from the voltage to the spool process are ignored in this paper.

\section{Design Example}

In this section, the proposed fuzzy modelled based adaptive robust controller is applied to the electrohydraulic suspension model of a full car. As mentioned earlier, following the conventional research method, we select three fundamental performance indexes, namely, the body acceleration, the suspension deflection, and the tyre dynamic displacement, as the evaluation quantified parameter to arrive at an assessment
TABLE 2: Structure parameters.

\begin{tabular}{lc}
\hline Parameter & Value \\
\hline$K_{f}=K_{r}$ & $16000 \mathrm{~N} / \mathrm{m}$ \\
$m$ & $1300 \mathrm{~kg}$ \\
$m_{i}(i=1,2,3,4)$ & $60 \mathrm{~kg}$ \\
$B_{f}=B_{r}$ & $1000 \mathrm{Ns} / \mathrm{m}$ \\
$k_{t i}(i=1,2,3,4)$ & $190000 \mathrm{~N} / \mathrm{m}$ \\
$a$ & $1.2 \mathrm{~m}$ \\
$c$ & $0.7 \mathrm{~m}$ \\
$I_{x x}$ & $550 \mathrm{~kg} \cdot \mathrm{m}^{2}$ \\
$k_{f}$ & 0 \\
$q_{1}$ & $1.70 \times 10^{-6} \mathrm{~m}^{4}$ \\
$q_{2}$ & $1.86 \times 10^{-6} \mathrm{~m}^{5} / \mathrm{sec}^{\mathrm{N}} \mathrm{N}^{1 / 2}$ \\
$\theta$ & $3.79 \times 10^{11} \mathrm{~N} / \mathrm{m}^{4}$ \\
$\theta_{m}$ & $2.25 \times 10^{11} \mathrm{~N} / \mathrm{m}^{4}$ \\
$\theta_{M}$ & $5.28 \times 10^{11} \mathrm{~N} / \mathrm{m}^{4}$ \\
$b$ & $1.4 \mathrm{~m}$ \\
$d$ & $0.8 \mathrm{~m}$ \\
$I_{y y}$ & $1842 \mathrm{~kg} \cdot \mathrm{m}^{2}$ \\
$\Pi_{M}$ & $0.1 \mathrm{~N} / \mathrm{m}^{4}$ \\
\hline
\end{tabular}

TABLE 3: Control parameters.

\begin{tabular}{lcccccccc}
\hline Parameter & $\gamma^{2}$ & $\varepsilon$ & $\kappa$ & $\rho$ & $\delta$ & $\sigma$ & $\eta$ & $c_{1}$ \\
\hline Value & 333 & 0.96 & 0.00016 & 0.0001 & 0.012 & 10000 & 10 & 100 \\
\hline
\end{tabular}

of this new method. For comparison, we utilize our previous research results on ordinary Backstepping active suspension control and passive suspension. The structural parameter values are listed in Table 2 and the controller parameters are shown in Table 3.

In this example, parameters $\rho$ and $\gamma^{2}$ corresponding to different fuzzy membership functions are selected as shown in Table 3. The algorithm for selecting parameters for this controller is based on the principle that the larger the selected value of $\rho$, the smaller the deflection in the suspension and vice versa. The same principle applies to $\gamma^{2}$.

Figures 3 and 4 show the responses of the fuzzy model based robust adaptive control, Backstepping control, and the passive suspension for a mild road input of $0.03 \mathrm{~m}$ and malevolent road input of $0.12 \mathrm{~m}$, respectively. In terms of ride comfort, under a small disturbance, the T-S fuzzy control performs almost the same as Backstepping, but better than passive suspension according to the acceleration results. Furthermore, under a malevolent disturbance, both T-S fuzzy control and Backstepping control are capable of reducing the suspension deflection and preventing the suspension from hitting the limit. Actually, under extremely hard road conditions, the body acceleration will become deteriorated sharply if there comes the limit hitting using the passive suspension. From the perspective of road holding, the 


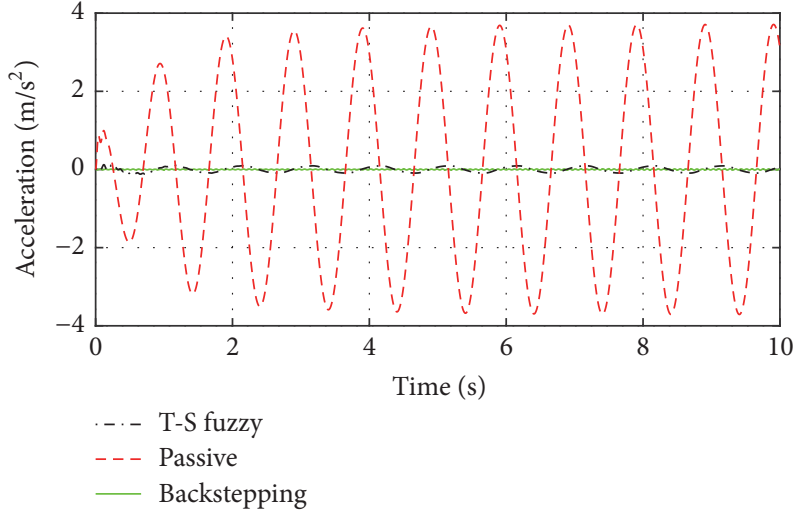

(a)

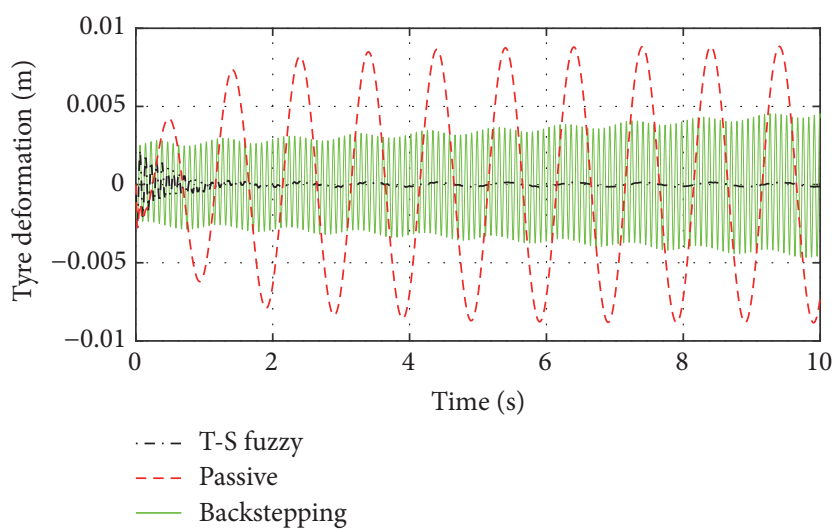

(c)

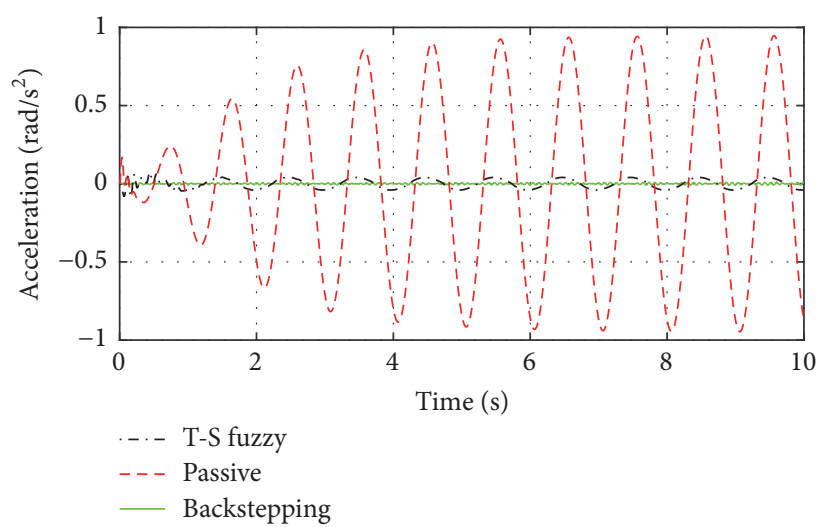

(e)

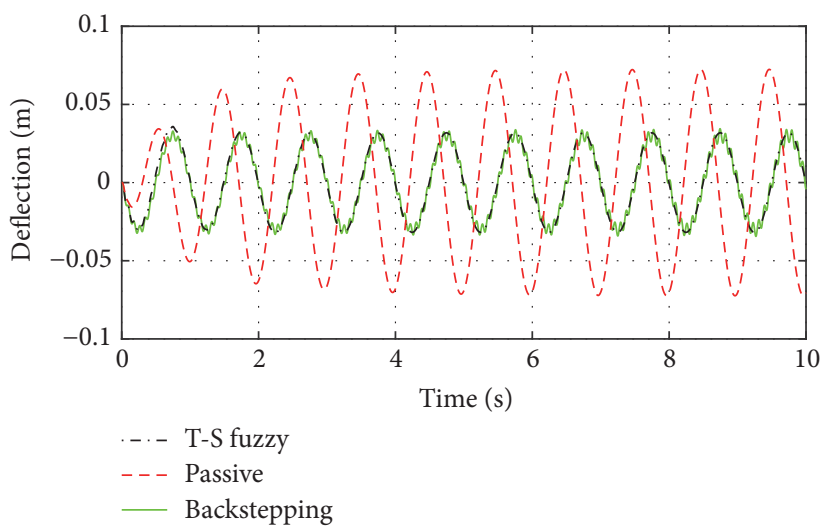

(b)

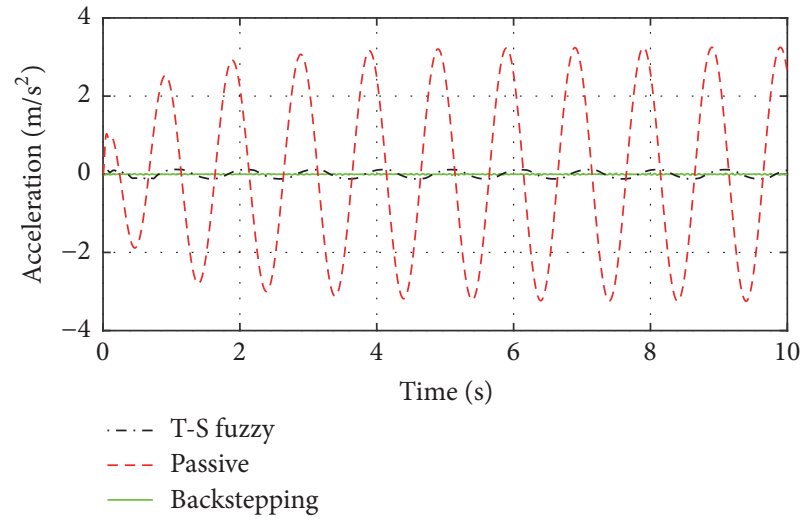

(d)

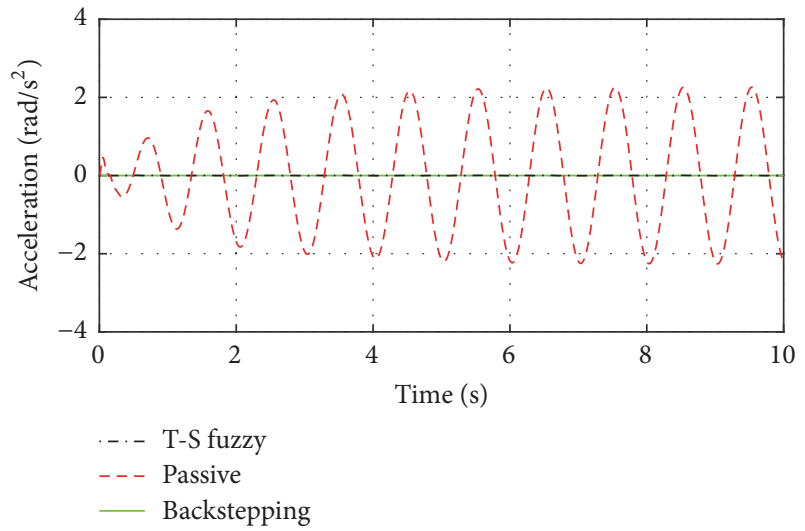

(f)

FIGURE 3: Output response for a mild road perturbation $(0.03 \mathrm{~m})$ : (a) acceleration response of front right corner; (b) dynamic deflection of front right corner; (c) tyre deformation of front right corner; (d) acceleration response of the center of mass; (e) acceleration response of the pitch motion; (f) acceleration response of the roll motion.

method used in this paper is not as excellent as Backstepping control as shown in Figures 3(c) and 4(c), since the constraint used in Backstepping control is stricter than the former control.

Figure 5 shows that, under a mild disturbance, the force produced from the Backstepping control is as large as that produced from the T-S fuzzy control. By contrast, under an intensive disturbance, T-S fuzzy control is capable of maintaining a considerably smaller force than Backstepping control while keeping the suspension from hitting the limit block. From these results, it follows that, since the Backstepping control objective is to maintain the displacement as close to zero as possible, the acceleration is strictly controlled in accordance with the control algorithm but without considering the energy efficiency. On the other hand, while using T$S$ fuzzy control under sustainable acceleration, the actuator 


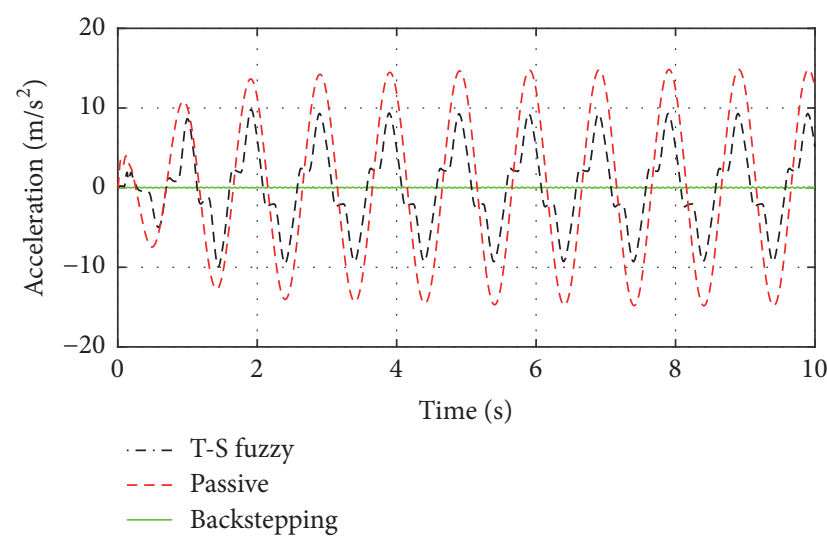

(a)

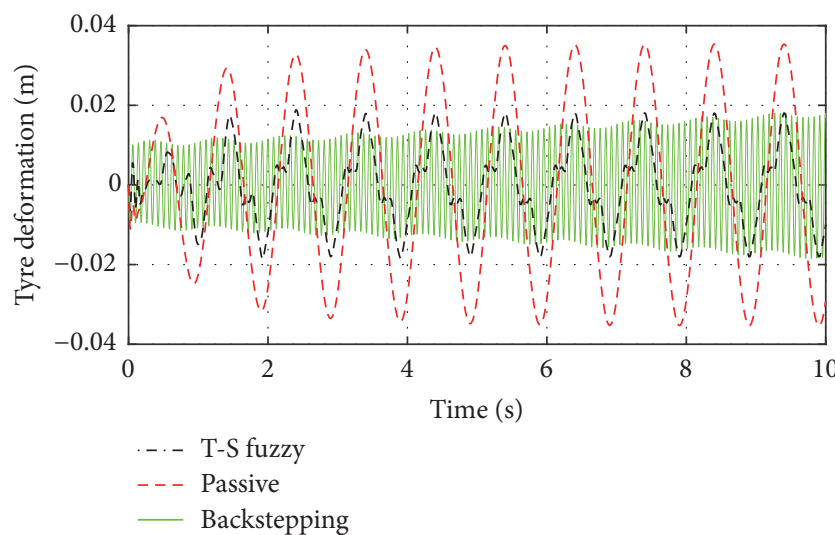

(c)

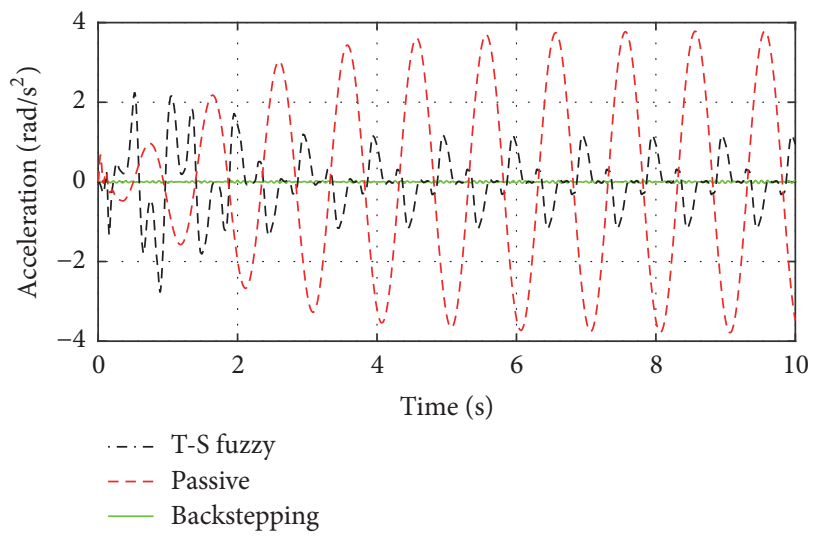

(e)

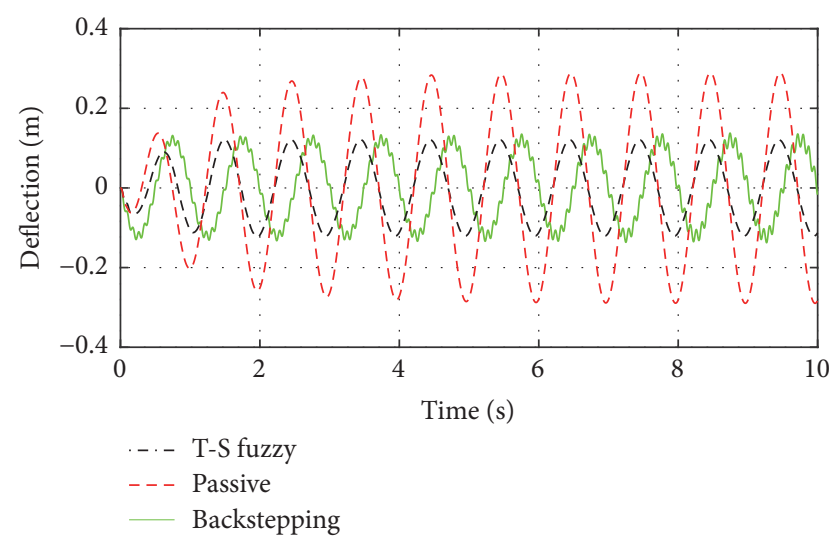

(b)

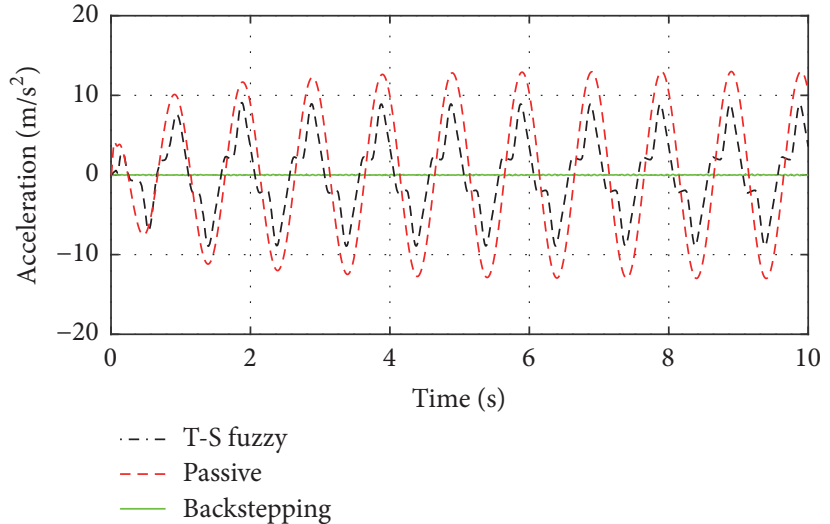

(d)

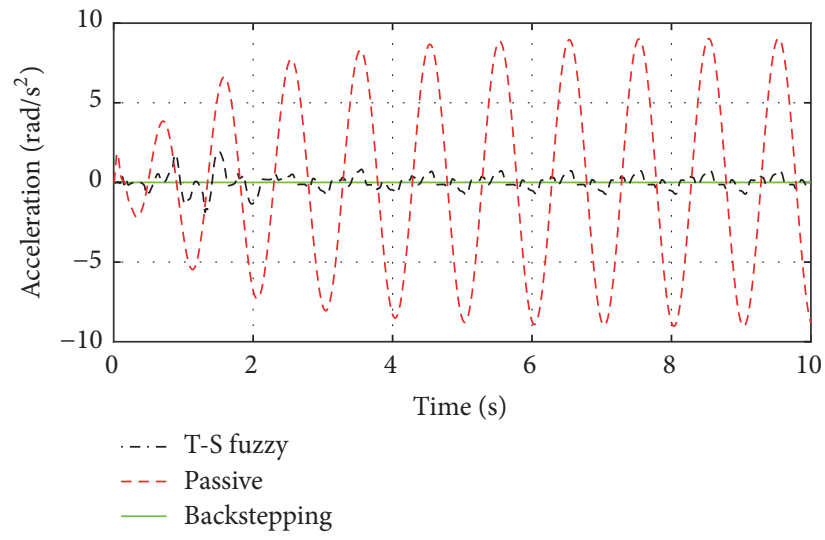

(f)

FIGURE 4: Output response for a malevolent road perturbation $(0.12 \mathrm{~m})$ : (a) acceleration response of front right corner; (b) dynamic deflection of front right corner; (c) tyre deformation of front right corner; (d) acceleration response of the center of mass; (e) acceleration response of the pitch motion; (f) acceleration response of the roll motion.

efficiency is taken into consideration even as the acceleration becomes larger.

Figure 6 shows tracking performance variations in robustness between the ARC control and the conventional Backstepping control as parameter $\theta$ is changed to $1.4 \theta$. It is seen that the adaptive robust control plays an important role in dealing with the uncertain nonlinearity compared to the conventional Backstepping active suspension. Here, we only take the parameter $\theta$ into consideration to verify robustness; that is, the nonlinearities related to frequency are not included in this paper, although it is acknowledged that it is an important issue for future research.

Figure 7 shows the frequency properties of inner loop system whose input is desired force and output is actual force. 


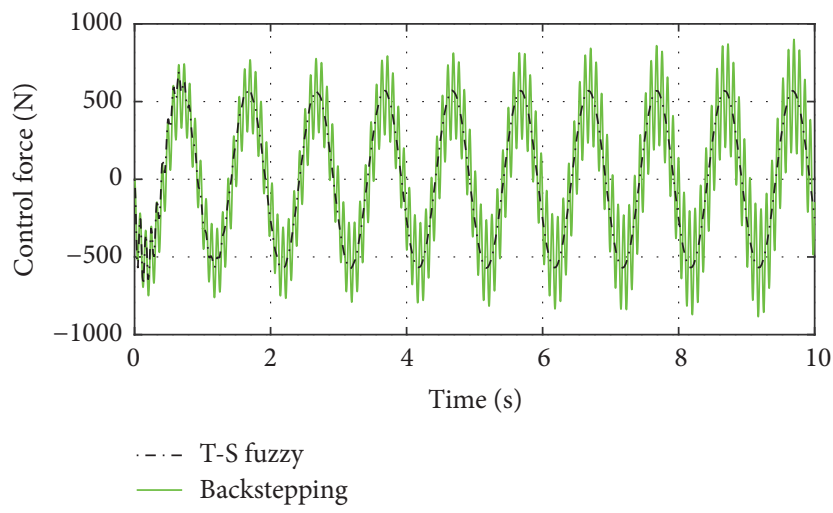

(a)

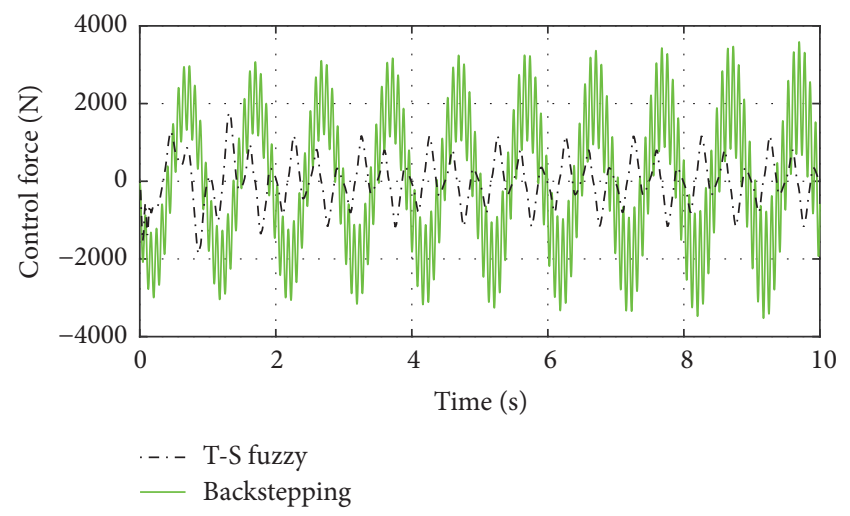

(b)

FIGURE 5: Force produced by the actuator: (a) force produced under mild perturbation; (b) force produced under malevolent perturbation.
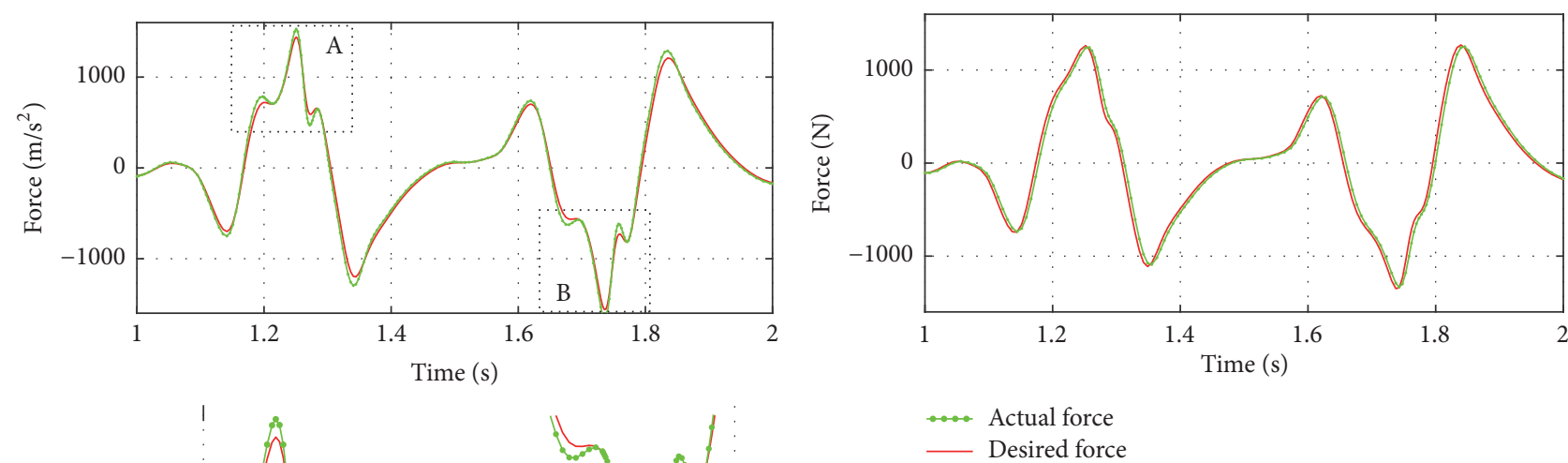

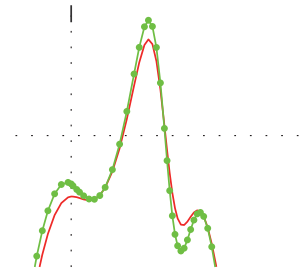

A

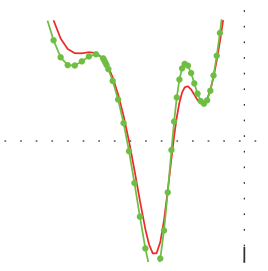

B

(a)

(b)

FIGURE 6: Tracking performance of the inner loop control: (a) T-S fuzzy based Backstepping control; (b) T-S fuzzy based ARC control.

It is shown that the ARC method can keep more favourable tracking property than ordinary Backstepping control. When the sprung mass changes from $1300 \mathrm{~kg}$ to $1500 \mathrm{~kg}$, the tracking performance can still be steadily held. Also, in the bandwidth from $0.1 \mathrm{~Hz}$ to $10 \mathrm{~Hz}$, superior tracking property using ARC method can always be possessed.

\section{Conclusion}

An active suspension using T-S fuzzy control and ARC control has been designed for a 7-DoF full car model. Unlike most previous 7-DoF full car models, the proposed full car model has been found to be capable of designing the separate control force on each corner. A fuzzy method consisting of
16 fuzzy rules is employed to form the performance output matrix, such that the aim to reduce the body acceleration is directly integrated into the LMIs resolution problem. The optimal feedback gains can be derived from these inequalities which consist of different control objectives, such as better ride comfort and vehicle handling. Moreover, the T-S fuzzy control tunes the fuzzy parameter according to the road input, which makes the output control force depend on the road disturbance, so that the actuator's efficiency is improved under the suspension rattle space constraint. The ARC control used in the inner loop is employed to preserve the robust stability. From the example results, this proposed active suspension holds better efficiency compared with ordinary Backstepping control, though both of them supply 

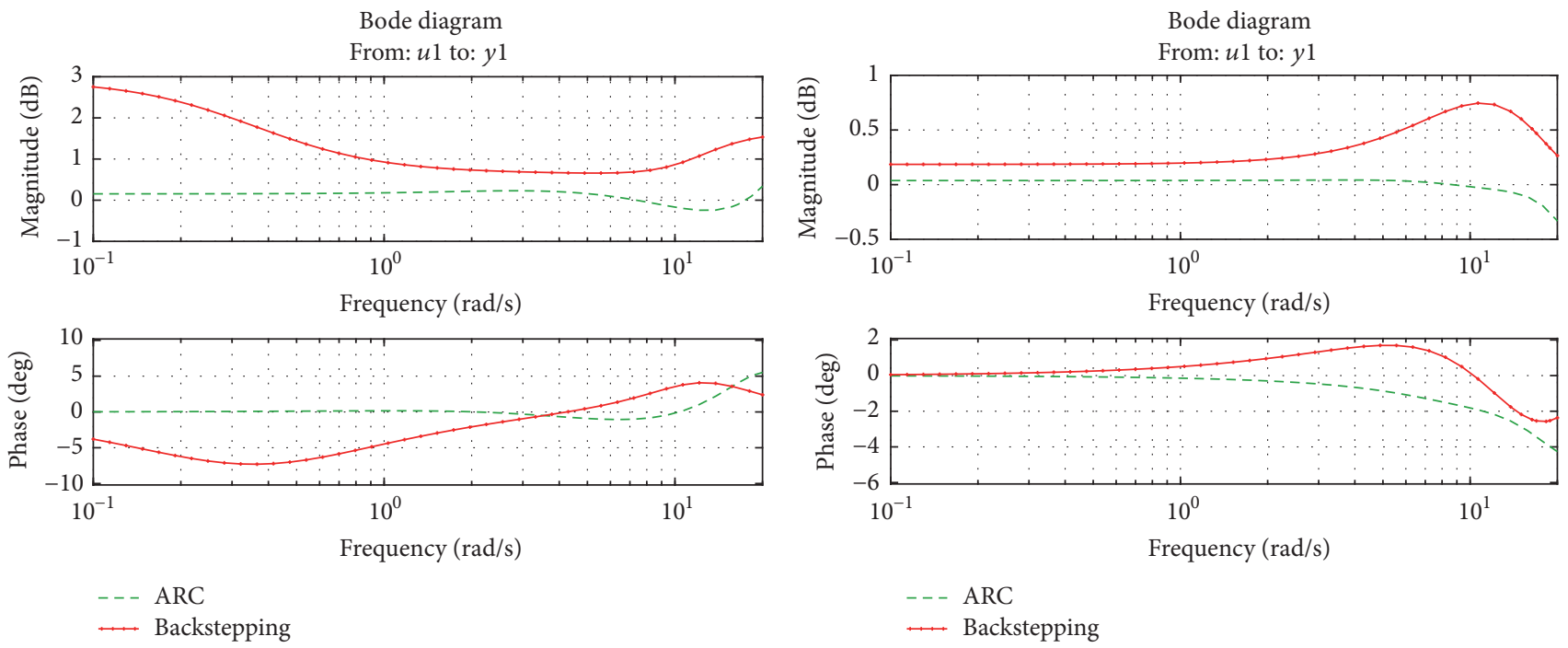

(a)

(b)

Figure 7: Tracking performance of different mass: (a) $m=1300 \mathrm{~kg}$; (b) $m=1500 \mathrm{~kg}$.

the favourable suspension properties. The ARC method plays an important role in keeping better tracking performance in the frequency bandwidth ranging from $0.1 \mathrm{~Hz}$ to $10 \mathrm{~Hz}$ and preserving the robustness effectively. In order to improve the performance of active suspension under different frequency, the response in frequency domain will be researched further with more parameters changing.

\section{Conflicts of Interest}

The authors declare that they have no conflicts of interest.

\section{Acknowledgments}

This work was supported by the Research Fund for the Doctoral Program of Higher Education (no. 20110205110008). The authors wish to thank the Automobile College for supporting the tests and construction involved. Thanks are also due to the group researching vehicle engineering at Chang'an University.

\section{References}

[1] T. P. J. Van Der Sande, B. L. J. Gysen, I. J. M. Besselink, J. J. H. Paulides, E. A. Lomonova, and H. Nijmeijer, "Robust control of an electromagnetic active suspension system: Simulations and measurements," Mechatronics, vol. 23, no. 2, pp. 204-212, 2013.

[2] Z. Li, L. Zuo, G. Luhrs, L. Lin, and Y.-X. Qin, "Electromagnetic energy-harvesting shock absorbers: design, modeling, and road tests," IEEE Transactions on Vehicular Technology, vol. 62, no. 3, pp. 1065-1074, 2013.

[3] X. Su, X. Yang, P. Shi, and L. Wu, "Fuzzy control of nonlinear electromagnetic suspension systems," Mechatronics, vol. 24, no. 4, pp. 328-335, 2014.
[4] M. S. Kumar and S. Vijayarangan, "Analytical and experimental studies on active suspension system of light passenger vehicle to improve ride comfort," Mechanics, vol. 65, no. 3, pp. 34-41, 2016.

[5] N. Yagiz and Y. Hacioglu, "Backstepping control of a vehicle with active suspensions," Control Engineering Practice, vol. 16, no. 12, pp. 1457-1467, 2008.

[6] W. Sun, H. Pan, Y. Zhang, and H. Gao, "Multi-objective control for uncertain nonlinear active suspension systems," Mechatronics, vol. 24, no. 4, pp. 318-327, 2014.

[7] A. Alleyne and R. Liu, "On the limitations of force tracking control for hydraulic servo-systems," Journal of Dynamic Systems, Measurement, and Control, vol. 121, no. 2, pp. 184-190, 1999.

[8] Y. Wang, L. Cao, S. Zhang, X. Hu, and F. Yu, "Command filtered adaptive fuzzy backstepping control method of uncertain nonlinear systems," IET Control Theory and Applications, vol. 10, no. 10, pp. 1134-1141, 2016.

[9] A. Goyal, J. Kumar, V. Kumar, and K. P. S. Rana, "Comparative study of BLF and QLF based backstepping controllers for active suspension system," in Proceedings of the 39th National Systems Conference, NSC 2015, Noida, India, December 2015.

[10] V. S. Deshpande, B. Mohan, P. D. Shendge, and S. B. Phadke, "Disturbance observer based sliding mode control of active suspension systems," Journal of Sound and Vibration, vol. 333, no. 11, pp. 2281-2296, 2014.

[11] Y. Feng, F. Han, and X. Yu, "Chattering free full-order slidingmode control," Automatica. A Journal of IFAC, the International Federation of Automatic Control, vol. 50, no. 4, pp. 1310-1314, 2014.

[12] B. Yao and M. Tomizuka, "Adaptive robust control of SISO nonlinear systems in a semi-strict feedback form," Automatica. A Journal of IFAC, the International Federation of Automatic Control, vol. 33, no. 5, pp. 893-900, 1997.

[13] J. Yao, Z. Jiao, and B. Yao, "Nonlinear adaptive robust backstepping force control of hydraulic load simulator: Theory and experiments," Journal of Mechanical Science and Technology, vol. 28, no. 4, pp. 1499-1507, 2014. 
[14] A. Mohanty, S. Gayaka, and B. Yao, "An adaptive robust observer for velocity estimation in an electro-hydraulic system," International Journal of Adaptive Control and Signal Processing, vol. 26, no. 12, pp. 1076-1089, 2012.

[15] L. Lu and B. Yao, "Energy-saving adaptive robust control of a hydraulic manipulator using five cartridge valves with an accumulator," IEEE Transactions on Industrial Electronics, vol. 61, no. 12, pp. 7046-7054, 2014.

[16] W. Sun, H. Gao, and B. Yao, "Adaptive robust vibration control of full-car active suspensions with electrohydraulic actuators," IEEE Transactions on Control Systems Technology, vol. 21, no. 6, pp. 2417-2422, 2013.

[17] J. Meng, Q. Chen, and R. He, "Research on optimal control for the vehicle suspension based on the simulated annealing algorithm," Journal of Applied Mathematics, vol. 2014, Article ID 420719, 2014.

[18] H. Chen and K. Guo, "Constrained Ho control of active suspensions: an LMI approach," IEEE Transactions on Control Systems Technology, vol. 13, no. 3, pp. 412-421, 2005.

[19] F. J. D'Amato and D. E. Viassolo, "Fuzzy control for active suspensions," Mechatronics, vol. 10, no. 8, pp. 897-920, 2000.

[20] Y. Jiang and Z.-P. Jiang, "Computational adaptive optimal control for continuous-time linear systems with completely unknown dynamics," Automatica. A Journal of IFAC, the International Federation of Automatic Control, vol. 48, no. 10, pp. 2699-2704, 2012.

[21] H. Li, J. Yu, C. Hilton, and H. Liu, "Adaptive sliding-mode control for nonlinear active suspension vehicle systems using $\mathrm{T}$ S fuzzy approach," IEEE Transactions on Industrial Electronics, vol. 60 , no. 8 , pp. $3328-3338,2013$.

[22] C. Zhai, W. Liu, and L. Huang, "The feedback control strategy of the Takagi-Sugeno fuzzy car-following model with two delays," Journal of Control Science and Engineering, Article ID 4581428, Art. ID 4581428, 11 pages, 2016.

[23] Y. Li and S. C. Tong, "Command-filtered-based fuzzy adaptive control design for MIMO switched nonstrict-feedback nonlinear systems," IEEE Transactions on Fuzzy Systems, vol. 25, no. 3 , pp. 668-681, 2017.

[24] Y. Li, S. Tong, L. Liu, and G. Feng, "Adaptive output-feedback control design with prescribed performance for switched nonlinear systems," Automatica. A Journal of IFAC, the International Federation of Automatic Control, vol. 80, pp. 225-231, 2017.

[25] S. C. Tong, Y. M. Li, and S. Sui, "Adaptive fuzzy output feedback control for switched nonstrict-feedback nonlinear systems with input nonlinearities," IEEE Transactions on Fuzzy Systems, vol. 24, no. 6, pp. 1426-1440, 2016.

[26] S. C. Tong, Y. M. Li, and S. Sui, "Adaptive fuzzy tracking control design for SISO uncertain nonstrict feedback nonlinear systems," IEEE Transactions on Fuzzy Systems, vol. 24, no. 6, pp. 1441-1454, 2016.

[27] H. Du and N. Zhang, "Fuzzy control for nonlinear uncertain electrohydraulic active suspensions with input constraint," IEEE Transactions on Fuzzy Systems, vol. 17, no. 2, pp. 343-356, 2009.

[28] H. Du and N. Zhang, "Static output feedback control for electrohydraulic active suspensions VIA T-S fuzzy model approach," Journal of Dynamic Systems, Measurement and Control, Transactions of the ASME, vol. 131, no. 5, pp. 1-11, 2009.

[29] B. Yao and M. Tomizuka, "Adaptive robust control of MIMO nonlinear systems in semi-strict feedback forms," Automatica. A Journal of IFAC, the International Federation of Automatic Control, vol. 37, no. 9, pp. 1305-1321, 2001.
[30] S. Chantranuwathana and H. Peng, "Adaptive robust force control for vehicle active suspensions," International Journal of Adaptive Control and Signal Processing, vol. 18, no. 2, pp. 83-102, 2004. 


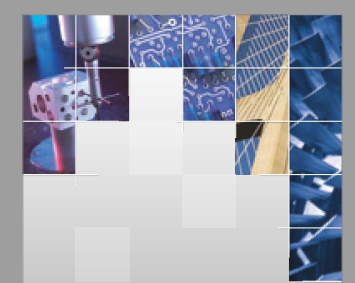

\section{Enfincering}
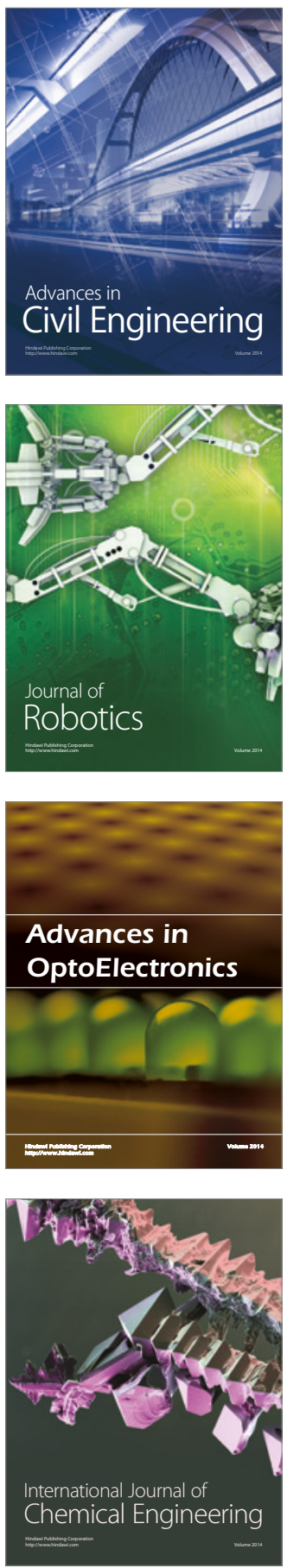

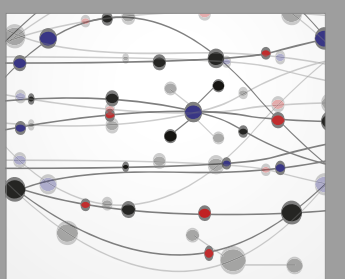

The Scientific World Journal

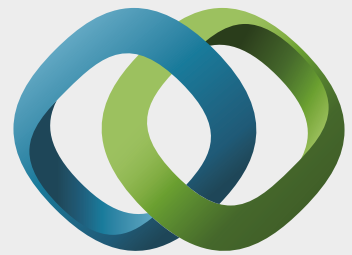

\section{Hindawi}

Submit your manuscripts at

https://www.hindawi.com
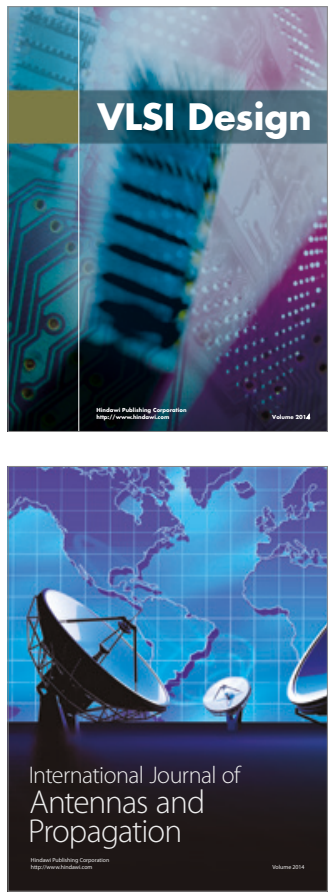

\section{Rotating}

Machinery
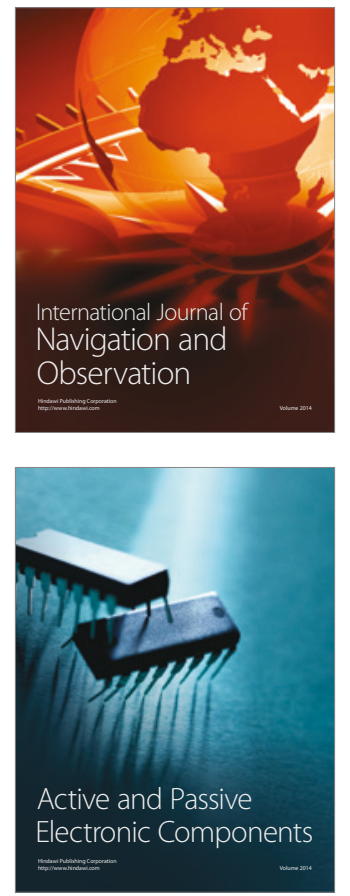
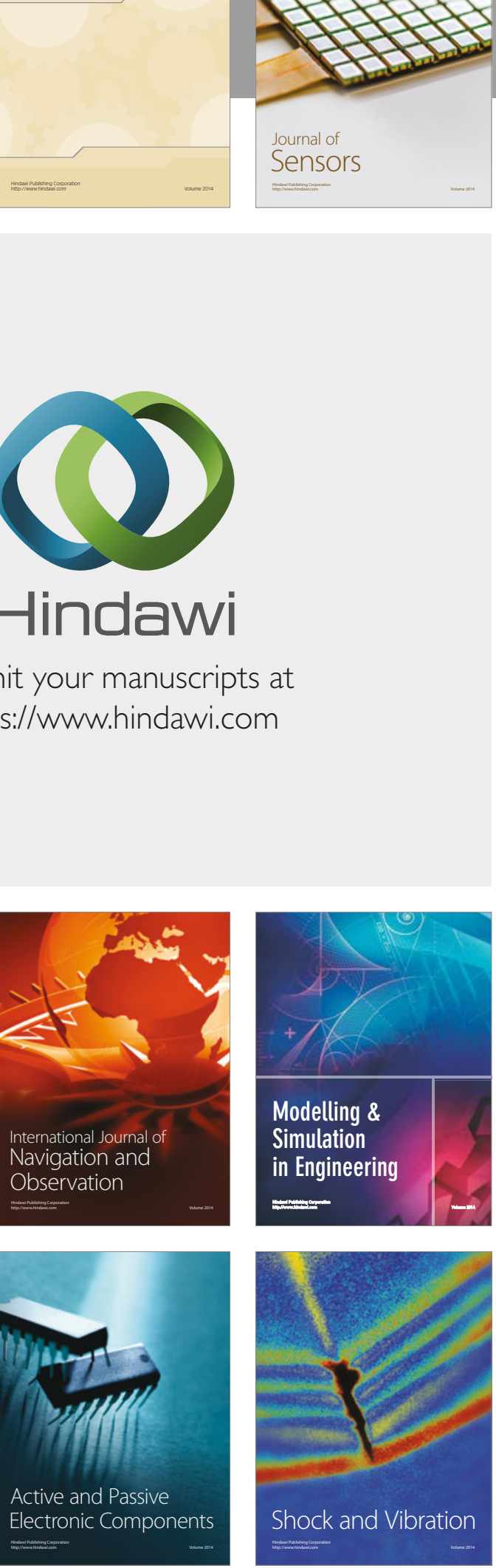
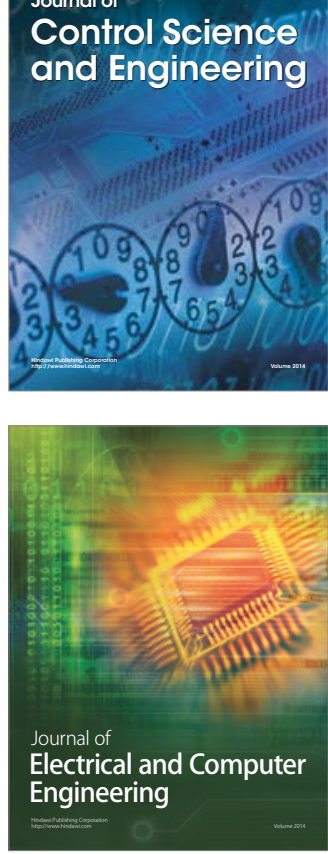

Distributed

Journal of

Control Science

and Engineering
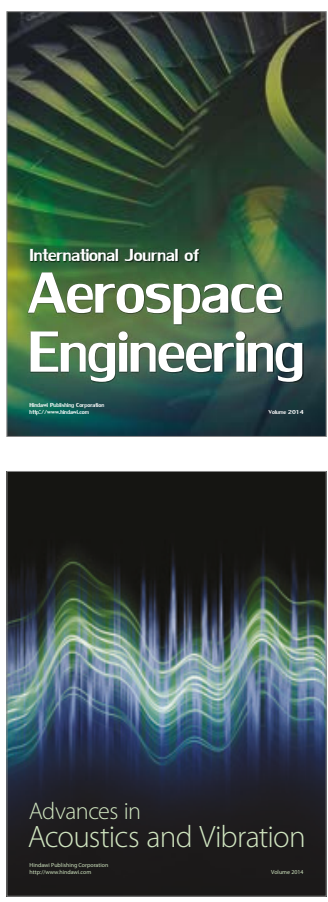

Sensor Networks 